\title{
Glucose uptake regulation in $E$. coli by the small RNA SgrS: comparative analysis of E. coli K-12 (JM109 and MG1655) and E. coli B (BL21)
}

\author{
Alejandro Negrete ${ }^{1}$, Weng-lan $\mathrm{Ng}^{1,2}$, Joseph Shiloach ${ }^{1 *}$
}

\begin{abstract}
Background: The effect of high glucose concentration on the transcription levels of the small RNA SgrS and the messenger RNA ptsG, (encoding the glucose transporter $\|_{C B}{ }^{\mathrm{Glc}}$ ), was studied in both E. coli K-12 (MG1655 and JM109) and E. coli B (BL21). It is known that the transcription level of sgrS increases when E. coli K-12 (MG1655 and $J M 109)$ is exposed to the non-metabolized glucose alpha methyl glucoside $(\alpha M G)$ or when the bacteria with a defective glycolysis pathway is grown in presence of glucose. The increased level of sRNA SgrS reduces the level of the ptsG mRNA and consequently lowers the level of the glucose transporter $\| \mathrm{CB}^{\mathrm{Glc}}$. The suggested trigger for this action is the accumulation of the corresponding phospho-sugars.

Results: In the course of the described work, it was found that E. coli B (BL21) and E. coli K-12 (JM109 and MG1655) responded similarly to $\alpha M G$ : both strains increased SgrS transcription and reduced pts $G$ transcription. However, the two strains reacted differently to high glucose concentration (40 g/L). E. coli B (BL21) reacted by increasing sgrS transcription and reducing ptsG transcription while E. coli K-12 (JM109 and MG1655) did not respond to the high glucose concentration, and, therefore, transcription of sgrS was not detected and ptsG mRNA level was not affected.

Conclusions: The results suggest that E. coli B (BL21) tolerates high glucose concentration not only by its more efficient central carbon metabolism, but also by controlling the glucose transport into the cells regulated by the sRNA SgrS, which may suggest a way to control glucose consumption and increase its efficient utilization.
\end{abstract}

\section{Background}

The currently preferred method for producing recombinant proteins from E. coli is to grow the bacteria to high cell density and consequently increase the volumetric productivity [1]. Growing the bacteria to high cell density is accomplished mainly by proper supply of dissolved oxygen and carbon source, usually glucose [2]. While implementing high density growth strategies, it was established that the central carbon metabolism in E. coli B (BL21) and E. coli K-12 (JM109) operates differently, especially when the bacteria are exposed to high glucose concentrations [3-6]. These differences are, at least in part, the result of a functioning glyoxylate shunt and high active gluconeogensis pathways in $E$. coli B (BL21) compared with E. coli K-12 (JM109). As a

\footnotetext{
* Correspondence: yossi@nih.gov

${ }^{1}$ Biotechnology Core Laboratory, NIDDK, NIH, Bethesda, MD USA

Full list of author information is available at the end of the article
}

result of these activities, E. coli $\mathrm{B}$ (BL21) tolerates higher glucose concentrations, produces less acetate, and grows to higher OD than E. coli K-12 (JM109) [5].

In the last several years, attention has been directed towards the small non-coding RNA (sRNA) in E. coli, and so far more than 70 sRNAs have been identified [7]. These molecules of approximately 40-400 nucleotides in length are involved in the regulation of pathways in response to stress conditions and environmental changes. The regulation occurs by altering translation or stability of mRNA and specific proteins through base pairing with target mRNAs or by binding to the proteins $[8,9]$.

One of the stress conditions studied was phosphosugars accumulation; in response to this accumulation, the mRNA ptsG, responsible for encoding the major glucose transporter, IICB ${ }^{\text {Glc }}$, was degraded [10-12]. The degradation occurs when the cells were exposed to the nonmetabolizable glucose analog alpha methyl glucoside

C 2010 Negrete et al; licensee BioMed Central Ltd. This is an Open Access article distributed under the terms of the Creative Commons Attribution License (http://creativecommons.org/licenses/by/2.0), which permits unrestricted use, distribution, and reproduction in any medium, provided the original work is properly cited. 
$(\alpha M G)$ [13], and also in response to the accumulation of glucose-6-phosphate (G6P) or fructose-6- phosphate in E. coli W3110 as a result of mutations in the glycolytic pathway $[11,12]$.

The molecule responsible for mRNA ptsG degradation was found to be the small RNA, SgrS, which was induced when the bacteria was exposed to $\alpha \mathrm{MG}$ or when mutation in the glycolytic pathway caused G6P accumulation $[11,13]$. The sRNA SgrS that caused the degradation of mRNA ptsG was also found to encode SgrT, a 43 amino acids polypeptide. This polypeptide rescues cells growing on $\mathrm{LB}$ media in the presence of $\alpha$ MG by inhibiting glucose transport even without mRNA degradation [14]. This phenomenon indicated physiological redundancy in response to phosphosugar accumulation. We suggest that the SgrS-ptsG mechanism may be activated not only in response to nonmetabolized glucose analog or to defective glycolytic enzymes, but also in response to high glucose concentration. Therefore, we decided to evaluate the response to high glucose in both E. coli B (BL21) and E. coli K-12 (JM109), two strains that are known to be different in their behavior when they are exposed to high glucose concentration [5]. If the assumption that acetate accumulation in $E$. coli grown at high glucose correlates with $s g r S$ transcription and biosynthesis of the glucose transporter is correct, then it might be possible to regulate the acetate excretion by controlling the sgrS transcription.

\section{Results}

Transcription of sgrS and ptsG in E. coli B (BL21) and E. coli K-12 (JM109, MG1165) growing in shake flasks in the presence of $20 \mathrm{~g} / \mathrm{L}$ glucose or $10 \mathrm{~g} / \mathrm{L} \alpha M G$

Previous studies of the role of SgrS and ptsG in E. coli metabolism were performed in shake flasks cultures; therefore the first phase of the present work was the evaluation of the differences between $E$. coli K-12 (MG1655 and JM109) and B (BL21) in shake flasks. The bacterial growth and the transcription of $s g r S$ and ptsG in E. coli B (BL21) and E. coli K-12 (MG1655 and JM109) on LB medium in presence of $\alpha M G$ or glucose in shake flasks are shown in Figures 1 and 2. After 2 hours growth $20 \mathrm{~g} / \mathrm{L}$ glucose or $10 \mathrm{~g} / \mathrm{L} \alpha \mathrm{MG}$ were added to the media (Addition indicated by the arrow in Figure 1). In the presence of $10 \mathrm{~g} / \mathrm{L} \alpha \mathrm{MG}$ E. coli K-12 (JM109) grew to an $\mathrm{OD}_{600}$ of 2 , while the other two strains grew to an $\mathrm{OD}_{600}$ of 4 (Figure 1a). When the three strains were grown in $20 \mathrm{~g} / \mathrm{L}$ glucose, the maximum $\mathrm{OD}_{600}$ value obtained was 6 for E. coli B (BL21) and 4 for the other two strains (Figure 1b). In the presence of glucose, cells reached stationary phase after 3 hours growth, which is the result of the decrease in $\mathrm{pH}$. The $\mathrm{pH}$ at the end of the run was around 4.5 for all strains. After six hours growth, the glucose concentration in the media of the three strains was around $10 \mathrm{~g} / \mathrm{L}$.

The corresponding sgrS and ptsG transcriptions following the addition of glucose or $\alpha M G$ are shown in Figure 2. When $\alpha M G$ was added to the medium, sRNA SgrS was observed in all three strains at all time points (Figure 2a), while mRNA ptsG appeared as faint bands. When the three strains were grown at a glucose concentration of $20 \mathrm{~g} / \mathrm{L}$, only E. coli B (BL21) showed transcription of $\operatorname{sgrS}$ (Figure 2b) whereas mRNA ptsG was detected in all strains. But when compared with $E$. coli K-12 (JM109 and MG1655) lower transcription of ptsG was observed in E. coli B (BL21). The internal controls SsrA and ompA were observed as uniform set of bands in all cases.

\section{Transcription of sgrS and ptsG in E. coli B (BL21) and E. coli K-12 (JM109, MG1165) growing in bioreactor in the presence of $40 \mathrm{~g} / \mathrm{L}$ glucose}

Following the shake flask growth, controlled growth of the three strains in a bioreactor at an initial glucose concentration of $40 \mathrm{~g} / \mathrm{L}$ was performed. The growth profiles and transcription of sgrS and ptsG in E. coli B (BL21) and E. coli K-12 (MG1655 and JM109) are shown in Figure 3 and 4. Cell growth, glucose consumption, acetate and pyruvate production are shown in Figure 3. All strains showed similar glucose consumption pattern: E. coli B (BL21) grew more than E. coli $\mathrm{K}$ -12 (MG1655 and JM109), acetate production from E. coli K-12 (MG1655 and JM109) was around $10 \mathrm{~g} / \mathrm{L}$ while $E$. coli B (BL21) produced only $4 \mathrm{~g} / \mathrm{L}$. Pyruvate concentration in E. coli K-12 (JM109) reached $15 \mathrm{~g} / \mathrm{L}$, compared with $2 \mathrm{~g} / \mathrm{L}$ in $E$. coli $\mathrm{K}-12$ (MG1655) and negligible concentration in E. coli B (BL21). The specific glucose consumption during the mid-log phase was $\sim 0.6$ gram per gram bacteria (wet weight) for $E$. coli B (BL21) and $E$. coli $\mathrm{K}-12$ (MG1655), and 1.3 gram per gram bacteria (wet weight) for E. coli K-12 (JM109). The corresponding transcriptions of $s g r S$ and $p t s G$ are shown in Figure 4. In E. coli K-12 (MG1655 and JM109) strains, no sRNA SgrS bands were detected during the first 3 hours of growth and faint bands were detected later; while higher transcription of SgrS was detected in E. coli B (BL21). The mRNA ptsG was observed in all strains, especially in the early growth stages, but lower transcription was observed in E. coli B (BL21). A decrease in the mRNA ptsG intensity was observed after 3 hours of growth, which may be due to the decreasing concentration of glucose in the media (Figure 3). The results obtained in the bioreactor were similar to those obtained in the shake flasks; the uniform detection of the internal controls SsrA and ompA in shake flasks and in the bioreactor indicated that the same amount of total RNA was loaded into the gel. 

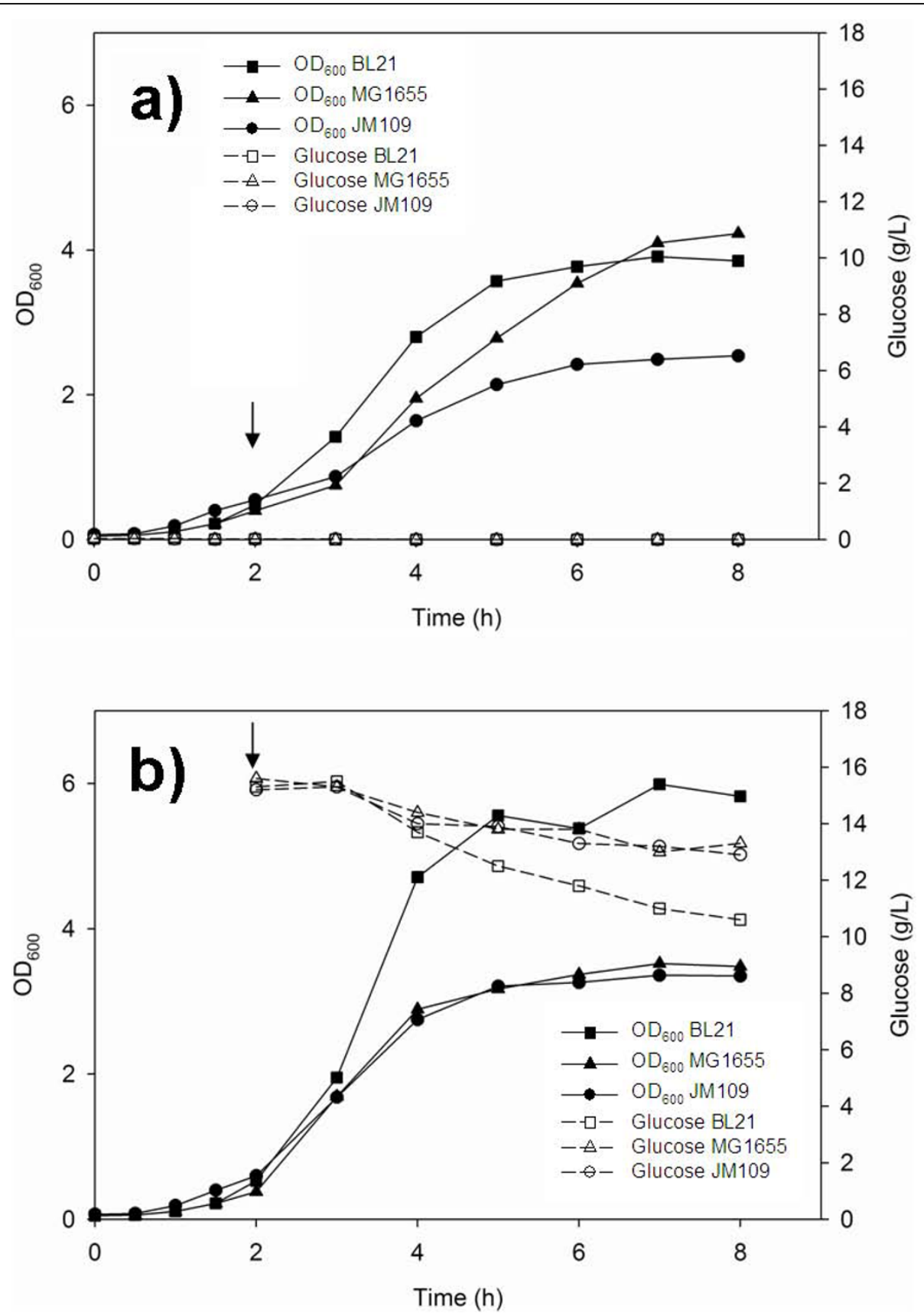

Figure 1 Growth parameters of $\boldsymbol{E}$. coli in shake flasks. Growth of $E$. coli in shake flask on: a) $10 \mathrm{~g} / \mathrm{L} \alpha$-methyl glucoside, b) $20 \mathrm{~g} / \mathrm{L}$ Glucose. Arrow indicates glucose or $\alpha M G$ addition.

Transcription of sgrS and ptsG in E. coli B (BL21) growing in the presence of low glucose in a bioreactor

As indicated in the introduction, E. coli B (BL21) is not affected by glucose concentration, its acetate excretion levels are the same whether the bacteria is exposed to high or low glucose concentration. The transcriptions levels of $\operatorname{sgrS}$ and $p t s G$ in this strain, growing in the presence of low glucose concentration, were measured to determine if the $\mathrm{SgrS}$ is involved in the control of the ptsG level. The results are seen in Figure 5. Comparing the transcription levels of $p t s G$ and $\operatorname{sgrS}$ when the media contained $40 \mathrm{~g} / \mathrm{L}$ glucose, with the transcription levels when the glucose concentration was kept around $1 \mathrm{~g} / \mathrm{L}$ throughout the growth, showed that $\operatorname{sgr} S$ transcription were lower and $p t s G$ transcription levels were higher. 


\section{a)}

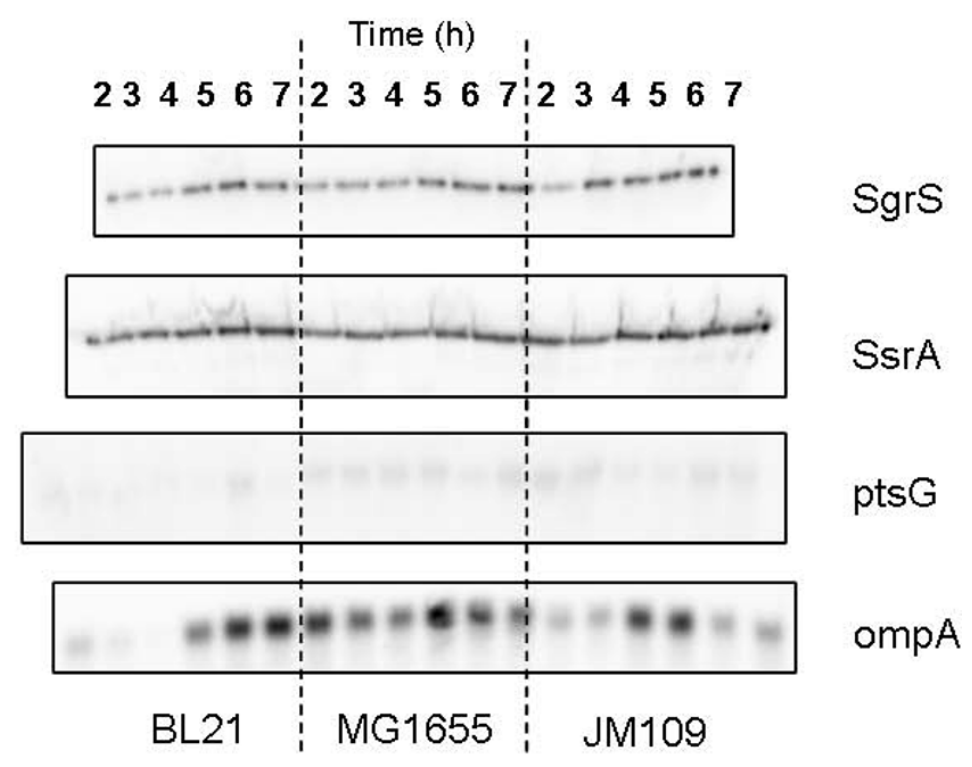

b)

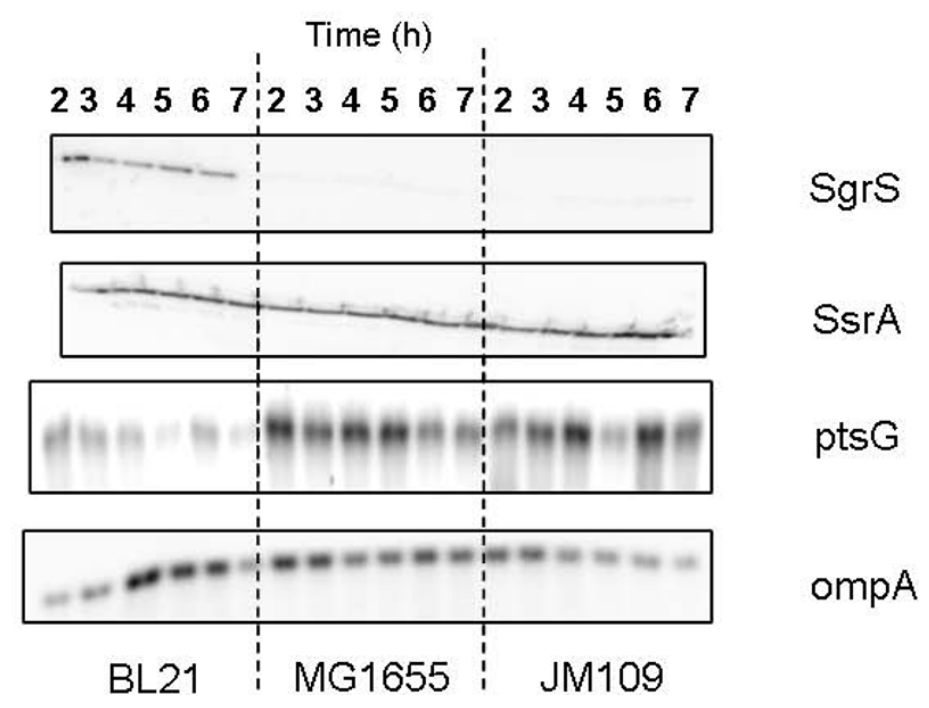

Figure 2 Transcription levels of sRNA and mRNA from $E$. coli growing in shake flasks. Transcription levels of sgrs, ssrA, ptsG, and ompA in E. coli B (BL21), E. coli K-12 (MG1655), and E. coli K-12 (JM109) grown in shake flask a) $10 \mathrm{~g} / \mathrm{L} \alpha$-methyl glucoside b) $20 \mathrm{~g} / \mathrm{L}$ glucose.

\section{Discussion}

Previous studies reported that increased levels of sRNA SgrS and reduced amounts of ptsG mRNA were observed in E. coli K-12 strains DJ480 PP6, IT1568, $\mathrm{DH} 5 \alpha$ and W3110 when the bacteria were grown in the presence of the non-metabolizing $\alpha \mathrm{MG}[11,13,15]$.
Reduced level of mRNA ptsG was also observed when E. coli $\mathrm{K}-12$ was grown on glucose when the glycolytic pathway was blocked by mutation in phosphoglucose isomerase or phosphofructokinase [11]. The sgrS transcription level was found to be correlated with the accumulation of $\alpha$ MG-phosphate or with G6P, and with the 

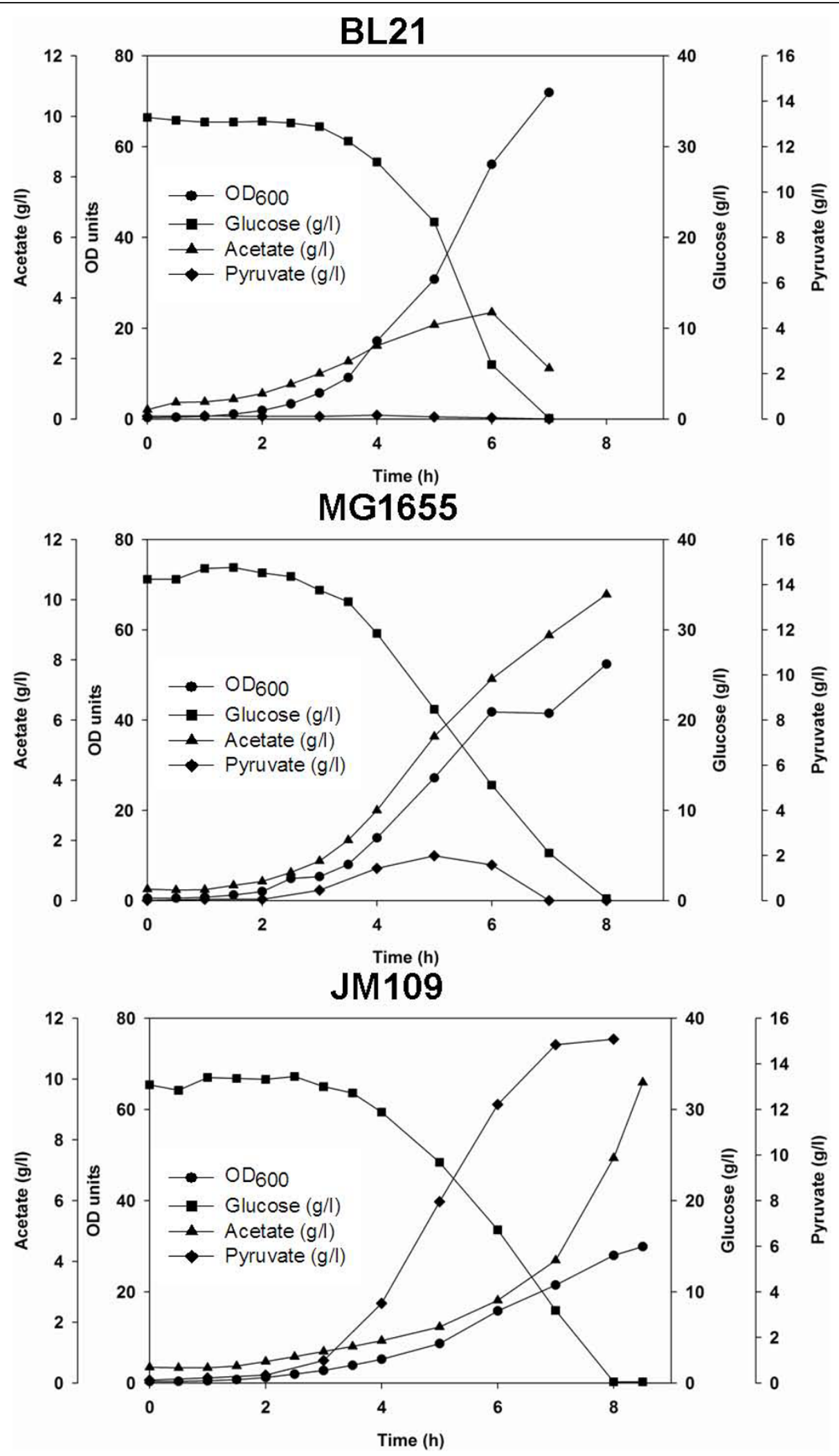

Figure 3 Growth parameters of E. coli in bioreactor. Growth of E. coli B (BL21), E. coli K-12 (MG1655) and E. coli K-12 (JM109) in a stirred tank bioreactor at $40 \mathrm{~g} / \mathrm{L}$ Glucose. 


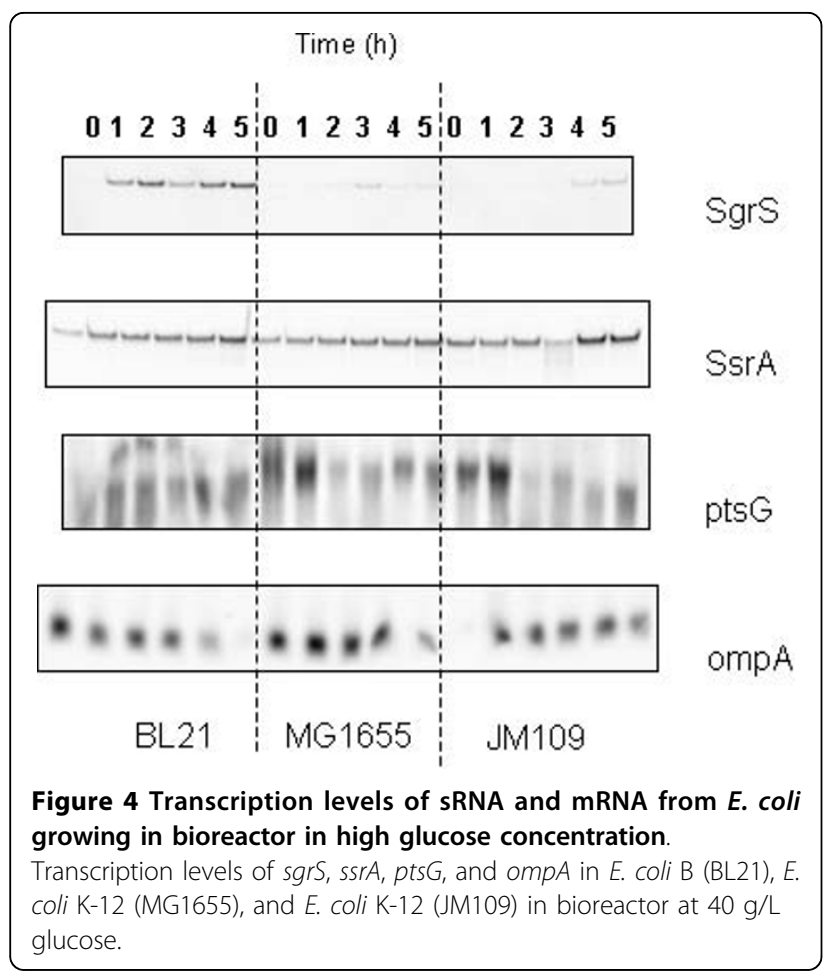

reduced level of the mRNA ptsG. The lower level of the mRNA ptsG was probably responsible for reduction in the biosynthesis of the glucose transporter $\mathrm{IICB}^{\mathrm{Glc}}$ $[13,16]$. When E. coli K-12, DH5 $\alpha$ and IT1568 were grown in the presence of $10 \mathrm{~g} / \mathrm{L}$ glucose, sRNA SgrS was not observed, while transcription of $p t s G$ was detected $[14,15,17]$.

The results presented in this work confirmed the previously reported $E$. coli $\mathrm{K}-12$ strains behavior in both shake flasks and in bioreactors, showing no sRNA SgrS

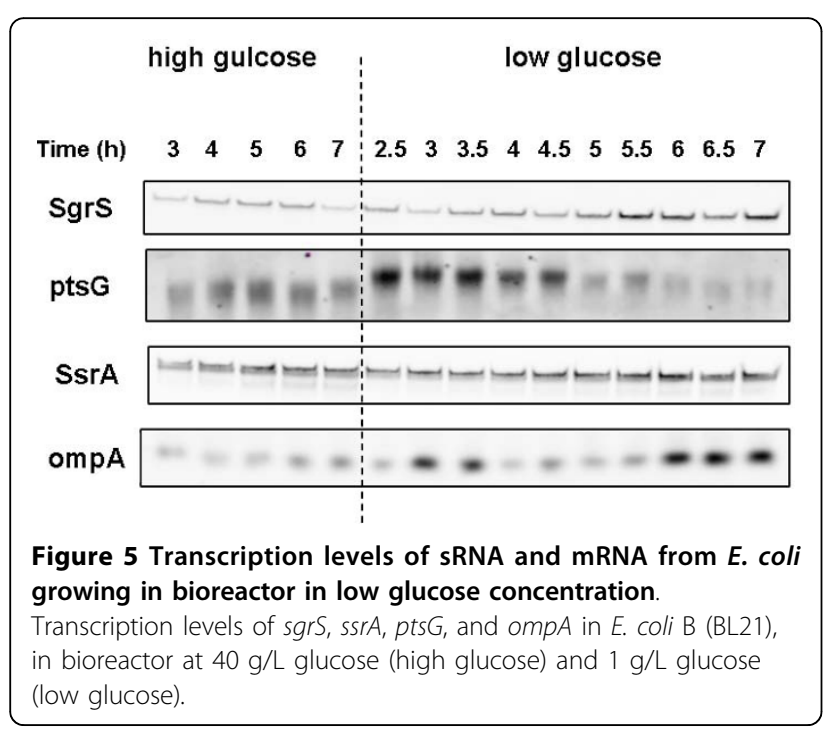

or significantly low levels even when the glucose concentration was as high as $40 \mathrm{~g} / \mathrm{L}$. E. coli B (BL21) behaved similarly to E. coli K-12 (MG1655 and JM109) strains when $\alpha \mathrm{MG}$ was added, but when the cells were exposed to $40 \mathrm{~g} / \mathrm{L}$ glucose, unlike the E. coli $\mathrm{K}-12$ (MG1655 and JM109) strains, sgrS was transcribed. When E. coli B (BL21) was grown in the presence of 1 $\mathrm{g} / \mathrm{L}$ glucose, the SgrS level was lower and the ptsG level was higher, increasing the glucose transport into the cells. It is possible that this mechanism together with CRP-cAMP and Mlc controls the expression of the glucose transporter $\mathrm{IICB}^{\mathrm{Glc}}$, thus reducing the internal glucose-phosphate concentration $[18,19]$. The E. coli B (BL21) responded to $40 \mathrm{~g} / \mathrm{L}$ of glucose concentration and to the presence of $\alpha \mathrm{MG}$ in the same way that E. coli K-12 (W3110) responded to $\alpha \mathrm{MG}$ and to glucose when the glycolytic pathway was blocked [12].

The acetate production pattern of the tested strains when grown on $40 \mathrm{~g} / \mathrm{L}$ glucose indicated better control of glucose assimilation in E. coli B (BL21) compared with E. coli K-12 (MG1655 and JM109) strains. This behavior is already known and is attributed to an active glyoxylate shunt, to higher flux through the TCA cycle, and to an active gluconeogensis pathway in E. coli $\mathrm{B}$ (BL21) [5]. The analysis of the SgrS-ptsG regulation suggests that the efficient intake of glucose and the lower production of pyruvate and acetate in E. coli B (BL21) are not only the result of different operation of the central carbon metabolism, but also the result of a different control of glucose transport into the cells. Since there is transcription of $s g r S$ in E. coli B (BL21) and there is no transcription of $\operatorname{sgrS}$ in $E$. coli K-12 (JM109 and MG1165), there is less ptsG in E. coli K-12 (JM109 and MG1165) than in E. coli B(BL21). The SgrS-ptsG regulation likely contributes to lower concentrations of glucose and glucose intermediates in the cells, avoiding overloading of the TCA cycle and the accumulation of pyruvate and acetyl-CoA that can lead to acetate production.

The $\operatorname{sgrS}$ was transcribed in both E. coli B (BL21) and E. coli K-12 (MG1655 and JM109) strains when $\alpha$ MG was present in the media. But when both strains were grown in high glucose concentration, sRNA SgrS was detected only in E. coli B (BL21). If the trigger for the sgrS transcription is the accumulation of $\alpha$ MG-phosphate or glycolytic intermediates such as G6P as described previously $[11,15]$, then it is possible that G6P or another intermediate of the glycolytic pathway accumulates in E. coli B (BL21) and does not accumulate in E. coli $\mathrm{K}-12$ (JM109) or in E. coli K-12 (MG1655). The presence of pyruvate in E. coli K-12 (JM109) may be an indication that there is no accumulation of G6P, which is metabolized by the glycolytic pathway. Pyruvate and acetate accumulate as a result of limited availability of 
acetyl CoA by the pta-ack pathway. The information concerning the glucose consumption by $E$. coli $\mathrm{B}$ (BL21) and $E$. coli K-12 (JM109) strengthens the hypothesis that the difference in the acetate excretion between these two strains is related to the difference in glucose consumption [20]. E. coli MG1655 behavior is different; like E. coli $\mathrm{K}-12$ (JM109), there is no $\operatorname{sgrS}$ transcription and there is acetate accumulation; but unlike E. coli K-12 (JM109), pyruvate accumulation is much lower [21]. It is possible that like in E. coli K-12 (JM109), G6P is not accumulated; rather it is being metabolized through the glycolytic pathway and contributes to the higher acetate concentration. It is more difficult to explain the E. coli K-12 (MG1655) behavior in response to pyruvate. It is also not certain that G6P is the trigger for the increase in the SgrS transcription in E. coli BL21 when it grows on high glucose concentration, as was the case when the glycolytic pathway was interrupted by mutation. It is possible that another intermediate or intermediates accumulate when E. coli B (BL21) grows at high glucose concentration and is responsible for the enhance transcription of the SgrS.

More work is required to understand the response of these microorganisms to the glucose concentration. Based on these results, our assumption is that the difference in the glucose transport is the result of the lower ptsG mRNA amount which contributes to lower concentration of the glucose transporter $\mathrm{IICB}^{\mathrm{Glc}}$. The control of the glucose transporter concentration is possibly another mechanism in E. coli B (BL21) which helps reducing the overflow of glucose and to minimize acetate production.

\section{Conclusions}

$\mathrm{SgrS}$ is a small RNA in E. coli which regulates the expression of the glucose transporter $\mathrm{IICB}^{\mathrm{Glc}}$ and hence the glucose uptake by the cells. sgr $S$ transcription was found to be regulated differently in E. coli B (BL21) and E. coli $\mathrm{K}-12$ (JM109 and MG1655) when the cells were exposed to high glucose concentration. In E. coli K-12, sgrS transcription was lower which contributes to higher transporter concentration and to higher glucose uptake. In E. coli B (BL21) the situation was the opposite; $\operatorname{sgrS}$ transcription was higher which leads to lower glucose transporter concentration and to lower glucose uptake. It was reported earlier that the reason for increased $\mathrm{sgrS}$ transcription may be related to glucose phosphates accumulation; this was shown when E. coli K-12 (JM109 and MG1655) was exposed to $\alpha$ MG or when the glycolysis pathway was blocked. We therefore suggest that the reason for the higher sgrS transcription in E. coli $\mathrm{B}$ (BL21) is related to accumulation of an intermediate or intermediates of the glycolytic pathway such as G6P which serves as an indication for a possible overflow of glucose. This situation is different in E. coli $\mathrm{K}-12$ (JM109 and MG1655) where, possibly, there is no accumulation of an intermediate of the glycolytic pathway and the glucose is converted to acetate and pyruvate. The above suggested mechanism contributes to the lower acetate production in E. coli B (BL21) when growing at high glucose concentration. The central carbon metabolism in E. coli B (BL21) is known to be more suitable to handle high glucose concentration as a result of its higher TCA flux and active gluconeogensis and glyxoyalte pathway. The control of the glucose transport into the cells is another tool in this bacterial strain to minimize the overflow of glucose and minimize acetate production.

\section{Materials and methods}

\section{$E$. coli strains and growth conditions}

E. coli K-12 (MG1655) (F-, $\lambda$-, ilvG-, rfb-50, rph-1); E. coli B BL21(DE3) (F-, ompT, hsdSB(rB-, mB-), gal, dcm (DE3)) and $E$. coli K-12 (JM109(DE3)) (endA1, recA1, gyrA96, thi, hsdR17(rk-, mk+), relA1, supE44, $\lambda-$-, _(lac-proAB), [F', traD36, proAB, laclqZ_M15], IDE3) (Promega Corp, Madison, WI) were used. Cells were grown at $37^{\circ} \mathrm{C}$ in modified LB medium containing $10 \mathrm{~g} / \mathrm{L}$ tryptone, $5 \mathrm{~g} / \mathrm{L}$ peptone, $5 \mathrm{~g} / \mathrm{L}$ yeast extract ( $15 \mathrm{~g} / \mathrm{L}$ for JM109), $5 \mathrm{~g} / \mathrm{L}$ $\mathrm{NaCl}$ and $5 \mathrm{~g} / \mathrm{L} \mathrm{K}_{2} \mathrm{HPO}_{4}$. After sterilization, $10 \mathrm{mM}$ $\mathrm{MgSO} 4$ and $1 \mathrm{ml} / \mathrm{L}$ trace metal solution were added. Overnight cultures were used to inoculate shake flasks at OD600 $=0.03$. Shake flasks were incubated at $37^{\circ} \mathrm{C}$ with constant agitation. When cells reached an OD600 of approximately $0.3,20 \mathrm{~g} / \mathrm{L}$ glucose or $1 \% \alpha \mathrm{MG}$ was added. After glucose or $\alpha \mathrm{MG}$ addition, samples were collected for sRNA and mRNA analysis. Cell culture was centrifuged at 13,000 g for $5 \mathrm{~min}$; the cell pellet and the supernatant for RNA extraction and metabolites analysis were maintained at $-80^{\circ} \mathrm{C}$. For bioreactor experiments, a 5 liters B. Braun fermentor equipped with data acquisition and adaptive dissolved oxygen control system was used. Following sterilization and supplement addition, the glucose concentration was adjusted to $40 \mathrm{~g} / \mathrm{L}$. $\mathrm{pH}$ was controlled at 7.0 by addition of $15 \%(\mathrm{w} / \mathrm{v}) \mathrm{NH}_{4} \mathrm{OH}$, and dissolved oxygen (DO) was controlled at $30 \%$ of air saturation. The bioreactor was inoculated with $\mathrm{OD}_{600}$ of 0.3 and samples were collected and processed as described for shake flasks. Cell density was determined by measuring OD600 with a Pharmacia Biotech Ultrospec 3000 UV/ Visible spectrophotometer. For the low glucose concentration experiment, glucose was maintained at $1 \mathrm{~g} / \mathrm{L}$ by using DO control [22].

\section{Metabolite analysis}

Glucose was determined by YSI 2700 SELECT Biochemistry Analyzer. Acetate and pyruvate were analyzed by HPLC, Hewlett Packard 1100 Series using Aminex 
resin-based HPX-87 H column (Bio-Rad). Separation conditions were as follows: wavelength $210 \mathrm{~nm}$; mobile phase $0.008 \mathrm{~N} \mathrm{H}_{2} \mathrm{SO}_{4}$, flow rate $0.6 \mathrm{~mL} / \mathrm{min}$, temp $35^{\circ} \mathrm{C}$ for acetate and $60^{\circ} \mathrm{C}$ for pyruvate. Three standards with concentrations of $0.1 \mathrm{~g} / \mathrm{L}, 1.0 \mathrm{~g} / \mathrm{L}$, and $10 \mathrm{~g} / \mathrm{L}$ were used for the calibration curve. The retention time for the pyruvate was $10 \mathrm{~min}$ and for the acetate $14 \mathrm{~min}$.

\section{RNA extraction}

For RNA extraction, the hot phenol method was used: cell pellets were resuspended in $0.5 \%$ SDS, $20 \mathrm{mM}$ $\mathrm{NaAc}$, and $10 \mathrm{mM}$ EDTA and extracted twice with hot acid phenol:chloroform followed by two extractions with phenol:chlorform isoamyl alcohol. Absolute ethanol was added and the extract was kept at $-80^{\circ} \mathrm{C}$ for $15 \mathrm{~min}$. After centrifugation at $14,000 \mathrm{~g}$ for $15 \mathrm{~min}$, the pellets were washed in $70 \%$ ethanol. RNA was air dried and resuspended in ultrapure water (KD medical USA). RNA was quantified using NanoDrop 1000 spectrophotometer (Thermofisher Scientific).

\section{Northern blot analysis}

Northern blot analyses to detect sRNA SgrS were performed as described previously [13]. $5 \mu \mathrm{g}$ of total RNA was run on a TBE $10 \%$ urea polyacrylamide gel and transferred to a positively charged nylon membrane. A 5'-biotinylated oligonucleotide SgrS-specific probe and Bright-Star Biodetect non-isotopic kit (Ambion, Inc.) were used for detection. For detection of mRNA ptsG, $5 \mu \mathrm{g}$ of total RNA was run on a $1.2 \%$ denaturing agarose gel and transferred to a positively charged nylon membrane [23]. After hybridization, the washed membranes were conjugated with streptavidin-alkaline phosphatase following the BrightStar Biodetect Kit (Ambion). Chemiluminscent signals were detected using the Fujifilm LAS-4000 imaging system. The image before the saturation point was recorded. The internal controls SsrA and ompA were detected by probing again the stripped membranes with boiling in $0.5 \%$ SDS. The sequences 5 ' to 3' of the probes used were:

sgrS (Bio)-GCAACCAGCACAACTTCGCTGTCGC GGTAAAATAGTG

ptsG (Bio)-CAGCCAGCTGAAATTCGCGGAACC GACGCCCAGCAG

ssrA (Bio)-CGCCACTAACAAACTAGCCTGATTAA GTTTTAACGCTTCA

ompA (Bio)-CCATTGTTGTTGATGAAACCAGTGT CATGGTACTGGGACCAGC

\section{List of abbreviations}

CRP-CAMP: transcriptional dual regulator; MLC: global regulator of sugar metabolism; OMPA: messenger RNA encoding outer membrane protein used as internal control; PTSG: gene encoding for the polypeptide ptsG; PTSG: polypeptide ptsG; SGRS: gene encoding for the sugar transport related small
RNA; SGRT: peptide SgrT; SGRS: sugar transport related small RNA; SRNA: small RNA; SSRA: small RNA encoding transcription unit used as internal control.

\section{Competing interests}

The authors declare that they have no competing interests.

\section{Authors' contributions}

AN participated in the design of study, fermentations and molecular analysis, analyzed the results and drafted the manuscript. WN participated in the fermentations and the molecular analysis. YS conceived the study, participated in its design and coordination, and in the writing of the manuscript. All authors read and approved the final manuscript.

\section{Acknowledgements}

Funding was provided by the Intramural program at the National Institute of Diabetes and Digestive and Kidney Diseases, National Institutes of Health. The authors would like to thank Prof. Nam Sun Wang for the academic support of Ms Weng, Dr. Young-Jin Son for his help in the metabolites analysis and Dr. N. Majdalani for his help in setting up the sRNA detecting method.

\section{Author details}

${ }^{1}$ Biotechnology Core Laboratory, NIDDK, NIH, Bethesda, MD USA. ${ }^{2}$ Department of Chemical and Biomolecular Engineering, University of Maryland, College Park, MD USA.

Received: 1 June 2010 Accepted: 28 September 2010 Published: 28 September 2010

\section{References}

1. Sivashanmugam A, Murray V, Cui C, Zhang Y, Wang J, Li Q: Practical protocols for production of very high yields of recombinant proteins using Escherichia coli. Protein Sci 2009, 18:936-948.

2. Gosset G: Improvement of Escherichia coli production strains by modification of the phosphoenolpyruvate:sugar phosphotransferase system. Microb Cell Fact 2005, 16:4-14.

3. van de Walle M, Shiloach J: Proposed mechanism of acetate accumulation in two recombinant $E$. coli strains during high density fermentation. Biotechnol Bioeng 1998, 57:71-78.

4. Noronha SB, Yeh HJ, Spande TF, Shiloach J: Investigation of the TCA cycle and the glyoxylate shunt in Escherichia coli BL21 and JM109 using (13) CNMR/MS. Biotechnol Bioeng 2000, 68:316-327.

5. Phue JN, Noronha SB, Hattacharyya R, Wolfe AJ, Shiloach J: Glucose metabolism at high density growth of $E$. coli $\mathrm{B}$ and $E$. coli $\mathrm{K}$ : differences in metabolic pathways are responsible for efficient glucose utilization in E. coli $\mathrm{B}$ as determined by microarrays and Northern blot analyses. Biotechnol Bioeng 2005, 90:805-820.

6. Phue JN, Kedem B, Jaluria P, Shiloach J: Evaluating microarrays using a semiparametric approach: application to the central carbon metabolism of Escherichia coli BL21 and JM109. Genomics 2007, 89:300-305.

7. Gottesman S: The small RNA regulators of Escherichia coli: roles and mechanisms. Annual Rev Microbiol 2004, 58:303-328.

8. Wassarman KM, Zhang A, Storz G: Small RNAs in Escherichia coli. Trends Microbiol 1999, 7:37-45

9. Waters LS, Storz G: Regulatory RNAs in bacteria. Cell 2009, 136:615-628.

10. Gottesman S: Micros for microbes: non-coding regulatory RNAs in bacteria. Trends Genet 2005, 21:399-404.

11. Kimata $K$, Tanaka $Y$, Inada $T$, Aiba $H$ : Expression of the glucose transporter gene, $p t s G$, is regulated at the mRNA degradation step in response to glycolytic flux in Escherichia coli. EMBO J 2001, 20:3587-3595.

12. Morita T, El-Kazzaz W, Tanaka Y, Inada T, Aiba H: Accumulation of glucose 6- phosphate or fructose 6-phosphate is responsible for destabilization of glucose transporter mRNA in Escherichia coli. J Biol Chem 2003, 278:15608-15614.

13. Vanderpool CK, Gottesman S: Involvement of a novel transcriptional activator and small RNA in post-transcriptional regulation of the glucose phosphoenolpyruvate phosphotransferase system. Mol Microbiol 2004, 54:1076-1089. 
14. Wadler CS, Vanderpool CK: A dual function for a bacterial small RNA: SgrS performs base pairing-dependent regulation and encodes a functional polypeptide. Proc Natl Acad Sci USA 2007, 104:20454-10459.

15. Kawamoto H, Morita T, Shimizu A, Inada T, Aiba H: Implication of membrane localization of target mRNA in the action of a small RNA: mechanism of post-transcriptional regulation of glucose transporter in Escherichia coli. Genes Dev 2005, 19:328-338.

16. Vanderpool CK: Physiological consequences of small RNA-mediated regulation of glucose-phosphate stress. Curr Opin Microbiol 2007, 10:146-151.

17. Morita T, Maki K, Yagi M, Aiba H: Analyses of mRNA destabilization and translational inhibition mediated by Hfq-binding small RNAs. Methods Enzymol 2008, 447:359-78.

18. Kimata $K$, Takahashi $H$, Inada T, Postma $P$, Aiba H: cAMP receptor proteincAMP plays a crucial role in glucose-lactose diauxie by activating the major glucose transporter gene in Escherichia coli. Proc Natl Acad Sci USA 1997, 94:12914-12919.

19. Kimata $\mathrm{K}$, Inada T, Tagami $\mathrm{H}$, Aiba HA: Global repressor (MIC) is involved in glucose induction of the $p t s G$ gene encoding major glucose transporter in Escherichia coli. Mol Microbiol 1998, 29:1509-1519.

20. Shiloach J, Rinas U: Glucose and acetate metabolism in E. coli-System level analysis and biotechnological applications in protein production process. In Systems biology and biotechnology of Escherichia coli. Edited by: Lee SY. Springer Science+Business Media B.V; 2009:377-400.

21. Dittrich CR, Bennett GN, San KY: Characterization of the acetateproducing pathways in Escherichia coli. Biotechnol Prog 2005, 21:1062-1067.

22. Shiloach J, Kaufman J, Guillard AS, Fass R: Effect of glucose supply strategy on acetate accumulation, growth, and recombinant protein production by Escherichia coli BL21 (lambda DE3) and Escherichia coli JM109. Biotechnol Bioeng 1996, 49(4):421-428.

23. Masse E, Gottesman S: A small RNA regulates the expression of genes involved in iron metabolism in Escherichia coli. Proc Natl Acad Sci USA 2002, 99(7):4620-5.

doi:10.1186/1475-2859-9-75

Cite this article as: Negrete et al:: Glucose uptake regulation in E. coli by the small RNA SgrS: comparative analysis of E. coli K-12 (JM109 and MG1655) and E. coli B (BL21). Microbial Cell Factories 2010 9:75.

\section{Submit your next manuscript to BioMed Central and take full advantage of:}

- Convenient online submission

- Thorough peer review

- No space constraints or color figure charges

- Immediate publication on acceptance

- Inclusion in PubMed, CAS, Scopus and Google Scholar

- Research which is freely available for redistribution 